\title{
Temporal Patterns in Chlamydia Repeat Testing and Positivity Rates in Massachusetts
}

\author{
Elizabeth C. Dee ${ }^{\star 1,2}$, Katherine K. Hsu², Benjamin A. Kruskal ${ }^{3}$, John T. Menchaca ${ }^{1}$, \\ Bob Zambarano ${ }^{4}$, Noelle Cocoros ${ }^{1}$, Brian Herrick ${ }^{5}$, Michelle D. Weiss ${ }^{5}$, Ellen Hafer, \\ Diana Erani ${ }^{6}$, Mark Josephson ${ }^{6}$, Jessica Young ${ }^{1}$ and Michael Klompas ${ }^{1,7}$
}

\begin{abstract}
'Department of Population Medicine, Harvard Medical School and Harvard Pilgrim Health Care Institute, Boston, MA, USA; ${ }^{2}$ Bureau of Infectious Disease and Laboratory Sciences, Massachusetts Department of Public Health, Boston, MA, USA; ${ }^{3}$ Atrius Health, Boston, MA, USA; ${ }^{4}$ Commonwealth Informatics, Waltham, MA, USA; ${ }^{5}$ Cambridge Health Alliance, Cambridge, MA, USA; ${ }^{6}$ Massachusetts League of Community Health Centers, Boston, MA, USA; ${ }^{7}$ Department of Medicine, Brigham and Women's Hospital, Boston, MA, USA
\end{abstract}

\section{Objective}

To evaluate current rates and temporal trends in adherence with national guidelines recommending chlamydia test-of-cure for pregnant females and test-of-reinfection for all patients.

\section{Introduction}

Sexually transmitted disease treatment guidelines have incrementally added repeat testing recommendations for Chlamydia trachomatis infections over time, including test-of-cure 3 to 4 weeks following completion of treatment for pregnant women and test-of-reinfection for all patients approximately 3 months after infection. However, few studies have investigated adherence to these recommendations and whether the evolution of guidelines have led to changes in repeat testing patterns over time.

\section{Methods}

The Electronic medical record Support for Public Health surveillance network (ESPnet) was leveraged to analyze electronic health record data for three independent practice groups serving approximately $20 \%$ of the Massachusetts population. We identified all cases with laboratory-confirmed Chlamydia trachomatis infections between 2010 and 2015 and evaluated the frequency, timing, and results of subsequent chlamydia tests in the following year.

\section{Results}

Between 2010 and 2015, 972 pregnant female cases, 10,309 non-pregnant female cases, and 4,973 male cases had a positive $C$. trachomatis laboratory result. Test-of-cure within 3-5 weeks following an index positive test occurred in $36.8 \%$ of pregnant females. Testof-reinfection within 2-4 months of an index test occurred in $39.2 \%$ of pregnant females, $17.9 \%$ of non-pregnant females, and $9.0 \%$ of males. There were no significant increases in test-of-cure or test-ofreinfection rates over the study period for any groups. Among cases with repeat tests, $15.9 \%$ of pregnant females, $14.6 \%$ of non-pregnant females, and $16.3 \%$ of males had at least one repeat positive result within one year of the index positive result.

\section{Conclusions}

Chlamydia test-of-cure and test-of-reinfection rates are low, with no evidence of improvement over time. There are substantial opportunities to improve adherence to chlamydia repeat testing recommendations.

\section{Keywords}

communicable disease; Electronic medical record; STD treatment guidelines; chlamydia

\section{*Elizabeth C. Dee}

E-mail: elizabeth_dee@harvardpilgrim.org 